\title{
Wetting, Prewetting and Superfluidity
}

\section{P. Taborek}

Received: 28 May 2009 / Accepted: 9 June 2009 / Published online: 1 July 2009

(C) The Author(s) 2009. This article is published with open access at Springerlink.com

\begin{abstract}
Experiments on adsorption and wetting of quantum fluids $\left({ }^{4} \mathrm{He}\right.$ and ${ }^{3} \mathrm{He}$ ) on weakly binding alkali metal substrates are reviewed. Helium on weak substrates can undergo a variety of phase transitions including wetting, prewetting, layering, and liquid-vapor transitions. Another characteristic feature of weak substrates is the absence of an immobile quasi solid layer which is present on all conventional strong substrates. Both the absence of the immobile layer and the interaction with surface phase transitions can strongly affect superfluid onset. The Kosterlitz-Thouless superfluid transition can terminate in either a critical endpoint where it meets a first order structural transition or a tricritical point where it meets critical point such as the prewetting critical point or the $2 \mathrm{D}$ liquid-vapor critical point.
\end{abstract}

Keywords Kosterlitz-Thouless $\cdot$ Helium $\cdot$ Cesium $\cdot$ Rubidium $\cdot$ Lithium

PACS 67.25.bh $\cdot 67.60 . \mathrm{gf} \cdot 68.08 . \mathrm{Bc} \cdot 68.15 .+\mathrm{e} \cdot 68.43 . \mathrm{h}$

\section{Introduction}

Wetting is a very general phenomenon that occurs whenever a bulk phase transition takes place near a wall. If two phases A, and B coexist in bulk, the chemical potentials and grand free energies of $\mathrm{A}$ and $\mathrm{B}$ are equal. $\mathrm{A}$ wall or interface produces an inhomogeneous potential which will couple to the two phases differently. The interactions with the wall will typically prefer one phase over another, and that phase will wet the wall. Typical examples of A and B include: two distinct phases of a single component system such as liquid and vapor or solid and liquid; a binary mixture of two immiscible liquids with different concentrations such as ${ }^{3} \mathrm{He}-{ }^{4} \mathrm{He}$ mixtures

P. Taborek $(\bowtie)$

Department of Physics and Astronomy, University of California, Irvine, CA 92697, USA

e-mail: ptaborek@uci.edu 
A)

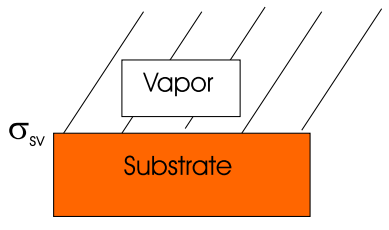

B)

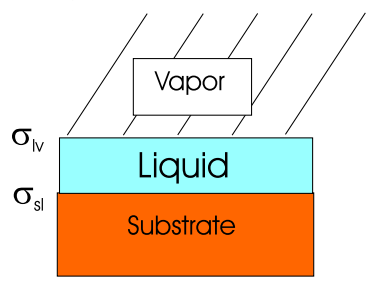

Fig. 1 (Color online) Schematic diagram of typical configurations corresponding to (A) the dry and (B) the wet case for a single component fluid near the liquid vapor transition. The grand free energy of each configuration must be evaluated to determine the stable state. $\sigma_{s v}, \sigma_{s l}$, and $\sigma_{l v}$, are the solid-vapor, solid-liquid and liquid-vapor surface tensions. Note that the wet case requires the formation of two interfaces, which is thermodynamically expensive when the surface tensions are large. Even more complicated layered structures need to be considered near triple points where three phases free energies

below the mixing critical point; and metallic phases with different magnetic or superconducting order [1]. Slightly away from coexistence, where in the thermodynamic limit only one phase could exist, the wall interactions can stabilize a microscopic layer of the less stable phase near the wall. This is what happens when, for example, a thin liquid film forms on an attractive wall in contact with a vapor below the saturated vapor pressure. The formation of such a wetting film requires the existence of an extra interface, as shown in Fig. 1. The cost of forming this interface must be compensated by a reduction in the total free energy of the system, due to a stronger interaction of the wetting phase with the wall. The structure of the film near the wall can be determined by computing the grand free energy for all possible configurations of phases, and picking the configuration that yields the lowest grand free energy at a given chemical potential. Near triple points, the wetting state can consist of more complicated stacks of layers with several interfaces [2]. The basic inputs required for this calculation are the interfacial energies and the substrate potential. A detailed discussion of the theoretical basis for a quantitative analysis is presented in [3] and [4].

\section{Wetting Transitions}

The global free energy minimum will depend on temperature because the bulk free energies, the densities and the interfacial tensions all depend on temperature. In particular, the liquid-vapor surface tension vanishes at the critical point, and has some large positive value at low temperatures, and varies monotonically and approximately linearly with temperature between these values. In most cases far from the critical point, the strongest temperature dependence is due to the surface tensions. Qualitatively, high temperatures imply low surface tensions, which favors the wet state, while at low temperatures, the cost of forming an extra interface can become prohibitive so the interface remains dry. On the other hand, if the surface potential is extremely strong, the wetting phase with the dense phase adjacent to the wall will always be preferred even at low temperatures. If the interactions of the fluid with the substrate is weaker or comparable to its interactions with itself, the competition between the wetting and nonwetting phases will be determined by the numerical value 


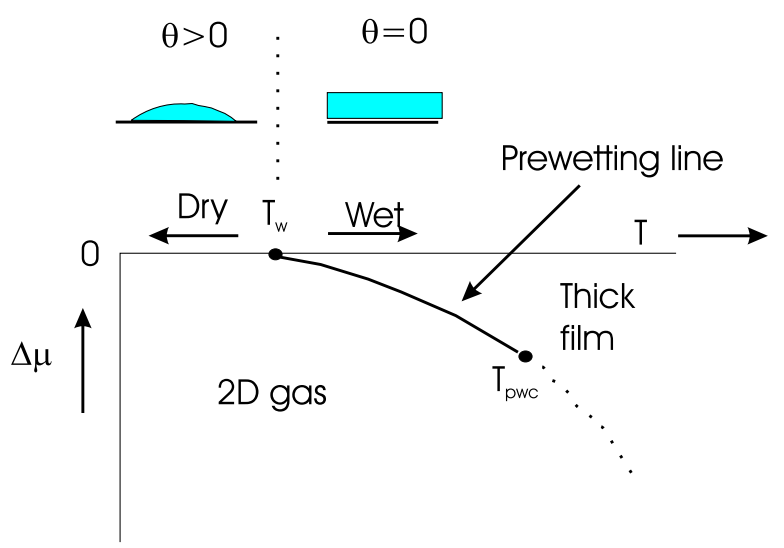

Fig. 2 (Color online) Generic prewetting phase diagram in the $\Delta \mu-T$ plane. $\Delta \mu$ is the difference from the bulk coexistence chemical potential, so the horizontal $\Delta \mu=0$ line represents liquid-vapor coexistence. At coexistence, the wetting temperature $T_{w}$ separates the wet and dry regions. If a drop of bulk liquid is placed on the surface below $T_{w}$, it forms a drop with a finite contact angle $\theta>0$. A drop placed on the wet side of the transition will spread indefinitely into a thin flat film with $\theta=0$. The prewetting line divides the plane into a "thin" 2D gas region and a "thick" region with a liquid film of finite thickness. The prewetting line extends from $T_{w}$ to the critical point $T_{p w c}$, but a region of high compressibility, denoted by the dotted line, extends much further into the plane

of the surface tensions, and will typically involve a first order wetting transition at $T=T_{w}$, with the substrate dry for $T<T_{w}$ and wet for $T>T_{w}$.

At bulk liquid-vapor coexistence, the wetting layer is macroscopically thick and the equilibrium film thickness has an essentially infinite jump in thickness as the temperature is raised from below to above $T_{w}$. If the wet state is approached at constant temperature by changing the chemical potential or the pressure, the film thickness again increases discontinuously across a line that emanates from $T_{w}$ in the $T-\mu$ plane. This curve is known as the prewetting line; it is a first order phase transition that separates a very thin 2D gas-like phase from a phase with a non-zero but finite thickness liquid film. Just as the analogous 3D liquid-vapor transition, the prewetting line ends in a prewetting critical point at $T=T_{p w c}$. A generic surface phase diagram for a weak substrate that illustrates these features is shown in Fig. 2.

The physics that generates the phase diagram of Fig. 2 is completely classical, but there are several experimental advantages to using cryogenic quantum fluids for investigating wetting phenomena. Helium in particular is chemically simple and pure, and the temperature in the cryogenic environment can be precisely measured and controlled. To exploit these advantages requires a weak substrate, i.e. a substrate with a helium interaction strength that is comparable to the helium-helium interaction. The traditional rule for estimating the interaction strengths involves the square root of the product of the polarizabilities of the constituent materials [5]. Helium has a very low polarizability and essentially everything else has a higher polarizability, so in this (naive) approximation, all walls are strong substrates, and we would expect helium to wet them even at $T=0$. This is corroborated by years of experience with helium adsorption on many conventional substrates such as gold, copper, glass, graphite, mylar, etc., which show no sign of a wetting transition. The naive approximation 
neglects the effects of the repulsive term in the potential. It was the great insight of Cheng, Cole, Saam, and Treiner [6] who realized that the short ranged repulsive part of the potential could have important effects on wetting. In particular, they made the surprising prediction that helium would not wet cesium. A qualitative explanation of this effect is based on the fact that cesium is the biggest atom in the periodic table, has the lowest ionization energy of any atom, and is extremely reactive. These properties reflect the fact that the outer electron of cesium is very weakly bound to the atom. At a solid metallic interface, the electrons in cesium extend anomalously far from the surface, which prevents helium from penetrating the deep part of the attractive long range potential and produces a very shallow bound state.

To experimentally verify this prediction requires producing an atomically clean and flat cesium surface and some means of detecting the surface coverage. Flatness is important because cracks and crevices can fill with liquid even if a flat surface would be dry; the associated effects of pore filling and capillary condensation are discussed elsewhere in this volume. The first experiments to explore wetting phenomena of helium on cesium used superfluid heat transport [7] and third sound [8] to detect the presence of a film. Our lab subsequently used quartz crystal microbalances (QCM) [9, 10] and ellipsometry [11], which can be used to detect both superfluid and normal adsorbed films. We evaporated approximately 80 layers of elemental cesium metal in situ onto the surfaces of a QCM and measured helium adsorption isotherms which were used to construct the first complete wetting phase diagram like that shown in Fig. 2. We found that for ${ }^{4} \mathrm{He}$ on $\mathrm{Cs}, T_{w} \approx 2.0 \pm 0.1 \mathrm{~K}$ and $T_{p w c} \approx 2.5 \mathrm{~K}$. We also found that at coexistence, the wetting transition was extremely hysteretic. If a dry state was prepared on the coexistence curve below $T_{w}$, it would spontaneously wet at $T=T_{w}$ as the temperature was raised. In contrast, a wet film prepared above $T_{w}$ would remain wet indefinitely even when it was cooled far below $T_{w}$. The basic reason for this asymmetry is that dewetting requires the nucleation of a dry patch, which in turn requires the formation of a large amount of liquid-vapor surface. On the coexistence curve, the nucleation barrier is essentially infinite, so the lifetime of the metastable wet film is also infinite [12].

The surface phases of other quantum fluids are also modified on weak substrates. ${ }^{3} \mathrm{He}$ wets Cs at all temperatures, but the formation of a thick film proceeds via an approximately first order prewetting-like transition [20]. The liquid-vapor surface tension of ${ }^{3} \mathrm{He}$ is less than for ${ }^{4} \mathrm{He}$, so the film state corresponding to Fig. $1 \mathrm{~B}$ is stable even at $T=0$. Hydrogen on $\mathrm{Cs}[13]$ and $\mathrm{Rb}$ [14] has a wetting and prewetting transition similar to Fig. 2. ${ }^{3} \mathrm{He}-{ }^{4} \mathrm{He}$ mixtures also have interesting behavior [15-18], including reentrant wetting, dewetting and prewetting [19], as illustrated in Fig. 3.

Another experimental method which can be used to locate the wetting transition is to measure the contact angle of a drop. It is complementary to the adsorption isotherm method because the contact angle grows continuously from $\theta=0$ at $T=T_{w}$ as the temperature is lowered from $T_{w}$. This type of measurement has been done for ${ }^{4} \mathrm{He}$ [21-23] and $\mathrm{H}_{2}$ [13] on Cs by taking optical photographs of drops through a long focal distance microscope. Despite the fact that the substrates are mirror-like surfaces with quite sharp prewetting steps, there is a very pronounced hysteresis between the advancing and receding contact angle. 


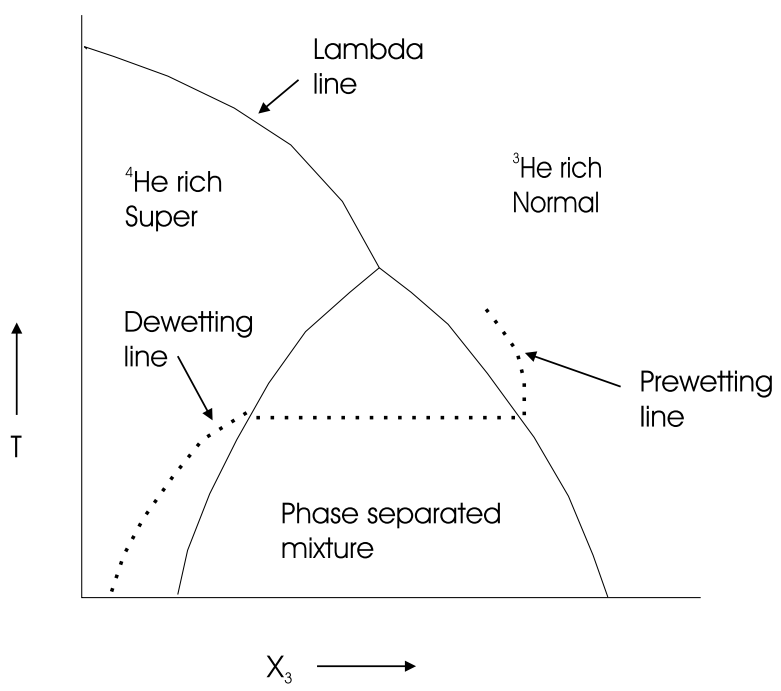

Fig. 3 Phase diagram of ${ }^{3} \mathrm{He}-{ }^{4} \mathrm{He}$ mixtures on cesium in the $T-X_{3}$ plane, where $X_{3}$ is the ${ }^{3} \mathrm{He}$ concentration. The bulk phase diagram has a region of ${ }^{4} \mathrm{He}$ rich liquid and a region of ${ }^{3} \mathrm{He}$ rich liquid which are separated by an inaccessible region of phase separated liquids which exists for temperatures below the mixing critical temperature $T_{m c}=0.87 \mathrm{~K}$. Below this critical temperature, the two liquids can coexist. The surface phase transitions are denoted by the dotted lines. On the ${ }^{4} \mathrm{He}$ rich side, there is a dewetting transition at temperatures below the wetting temperature $T_{w}=0.53 \mathrm{~K}$. The transition continues on the ${ }^{3} \mathrm{He}$ rich side in a prewetting transition. From [19]

Fig. 4 QCM signals for ${ }^{4} \mathrm{He}$ on gold, showing characteristic features of the KT transition. Frequency shift (solid squares) and resistance (open circles) as a function of the amount of helium added to the cell at $T=0.65 \mathrm{~K}$. The two near vertical steps in $\Delta f$ correspond to the formation of two solid layers. The KT transition is marked by a peak in the resistance and a sharp drop in $(-\Delta f)$

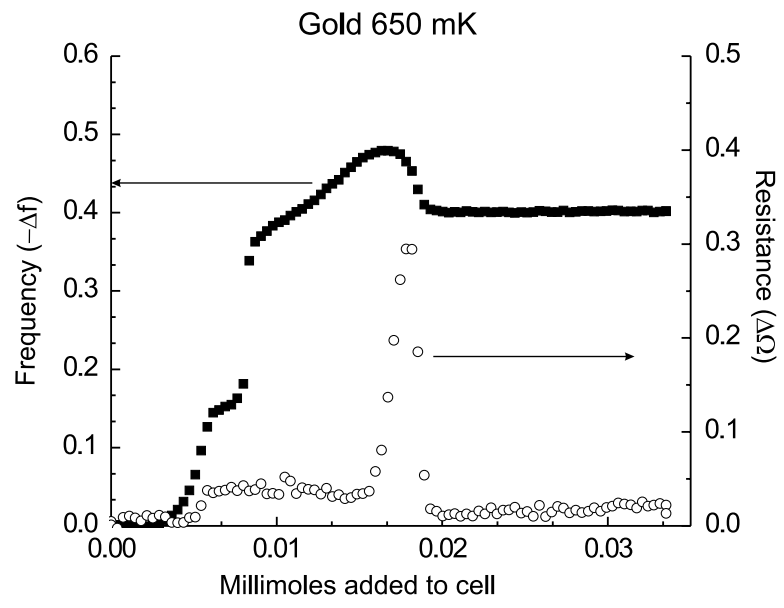

\section{Interactions with Superfluidity}

In addition to liquid and vapor phases, ${ }^{4} \mathrm{He}$ and dilute ${ }^{3} \mathrm{He}-{ }^{4} \mathrm{He}$ mixtures can also be either superfluid or normal. On a weak substrate, helium has a choice between thick, thin, superfluid and normal phases. In practice, the thin 2D gas phase is too dilute to support superfluidity, so there are 3 viable choices. As discussed above, the competition between thick and thin is determined by a balance of surface tensions and 
substrate potential, with a large substrate potential favoring a thick wet film, while a large liquid-vapor surface tension favors a thin dry state. The competition between super and normal is determined by a balance between the energy $E_{v}$ and the entropy $S_{v}$ of a vortex. In the simplest version of the Kosterlitz-Thouless (KT) theory of 2D phase transitions, a helium film will be superfluid until the free energy $F_{v}=E_{v}-T S_{v}$ is zero, when vortices will spontaneously form and destroy the long range order [24]. Both $E_{v}$ and $S_{v}$ are proportional to $\ln (A)$, where $A$ is the area of the film. $E_{v}$ is also proportional to the length of the vortex, i.e. the thickness of the film $d$. The supernormal transition occurs at a critical temperature $T_{K T}$, with $T_{K T} \propto d_{c}$, where $d_{c}$ is a critical thickness. A more refined version of the argument gives

$$
k_{B} T_{K T}=d_{c} \frac{\rho_{s} h^{2}}{8 \pi m^{2}}
$$

where $\rho_{s}$ is the superfluid mass density, and $m$ is the mass of a ${ }^{4} \mathrm{He}$ atom. The transition temperature does not depend in any obvious way on the substrate potential or the surface tension, so the two transitions are nominally decoupled, although the substrate potential and prewetting affects the film thickness, which in turn determines $T_{K T}$. Superfluid onset on weak and conventional strong substrates differ in other ways. On a strong substrate (essentially anything other than the alkalis), the first few layers of helium are immobile and a finite critical coverage is required for superflow. This disordered quasi solid layer is sometimes called a Bose glass [25, 26]. It is not simply an inert spectator, but rather plays a role in superfluid onset that is not understood in detail [27]. Theoretical calculations indicate, however, that on alkali metals, there is no solid-like layer and the film is entirely fluid and mobile [28-30], so the superfluid moves in direct contact with a metallic solid rather than a solid helium substrate. This difference can affect the core size and the kinetic energy of the vortices [31]. The alkali metals are also unusual substrates for macroscopic flows. For a superfluid drop on Cs, the contact line is the edge of the superfluid, which does not exist in any other system. Superfluid drops roll down an inclined cesiated plane with constant acceleration in a nondissipative way [32].

A standard method of measuring both the coverage and the superfluid transition is with mechanical oscillators, with kilohertz torsional oscillators and megahertz QCMs being the most popular. The total normal coverage is related to the frequency shift. The superfluid transition is marked by an increase in frequency due to decoupling of the superfluid component and a peak in the dissipation of the oscillator due to irreversible energy flow into the vortex plasma when the oscillator period is comparable to the time required for the vortices to diffuse by their mean separation. The assumptions underlying this interpretation are that the quasi-solid layer and/or the normal component is viscously locked to the substrate and that the superfluid phase is homogeneous or at least connected over macroscopic lengths. It is important to consider the possibility that these assumptions are violated for weak substrates. The possibility of slip in the solid is discussed in references [33,34] and the ramifications of superfluidity occurring in isolated puddles is discussed in references [35, 36]. A typical example of these standard experimental signatures for helium on a strong substrate (gold) are illustrated in Fig. 4. On a strong substrate, the film thickness is a monotonically increasing function of the chemical potential, or equivalently, the pressure, so 
Fig. 5 Phase diagram of ${ }^{4} \mathrm{He}$ on 4.2 layers of $\mathrm{Cs}$ on gold in the $P-T$ plane; $P / P_{0}$ represents the saturated vapor pressure. The circles show the positions of the prewetting step. For this Cs thickness, $T_{w}$ is approximately $1.5 \mathrm{~K}$. The triangles show the position of the KT transition. The KT transition ends on the prewetting line in a critical endpoint. Ts is the region of thick superfluid film and Tn is thick normal film. From [38]

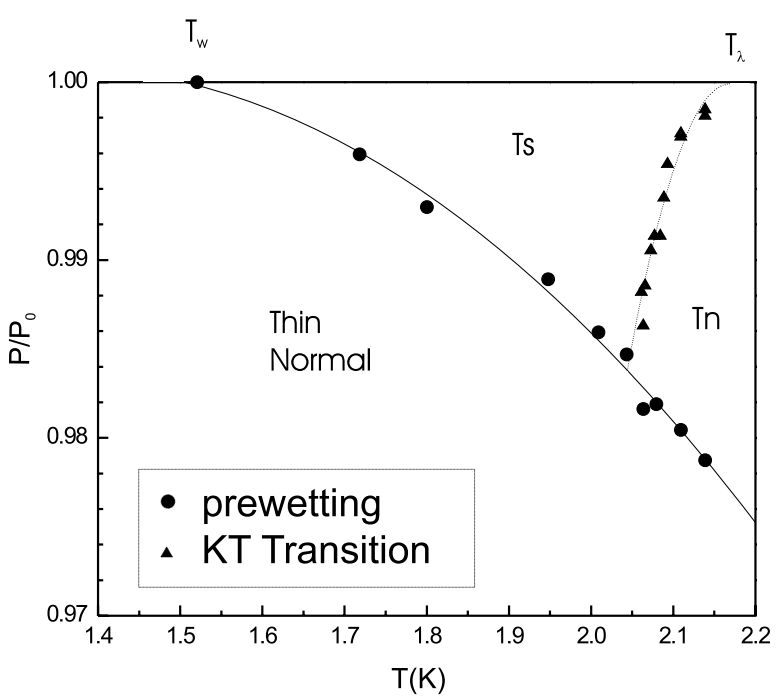

Fig. 6 Phase diagram of ${ }^{4} \mathrm{He}$ on rubidium in the $\Delta \mu-T$ plane; $\Delta \mu=0$ corresponds to bulk liquid-vapor coexistence. The open circles show the positions of the dissipation peak. The open triangles show the position of the steepest part of the prewetting step. The solid prewetting curve ends in a critical point marked by a solid dot. The dotted line is the KT transition line which separates the high temperature normal phase from the low temperature superfluid phase. The KT line terminates at the prewetting critical point, so it is a tricritical point. From [40]

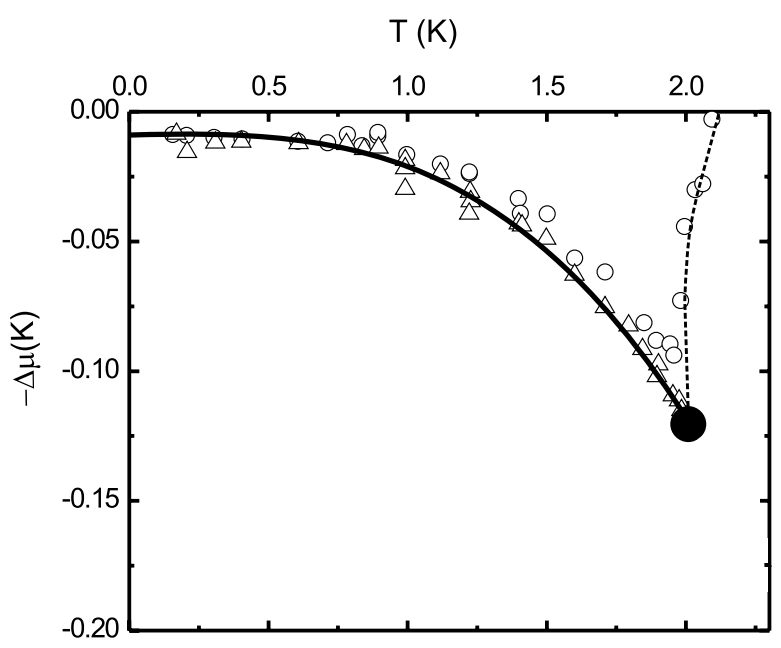

the combination of thickness and temperature required by (1) can be reached by either changing the coverage at constant temperature, as in Fig. 4, or by changing the temperature at constant coverage, as in [37].

On weak substrates, the interpretation of these experimental signatures requires more care. On (thick) cesium, the wetting temperature of ${ }^{4} \mathrm{He}$ is so high $(\sim 2.1 \mathrm{~K})$ and so close to the bulk $T_{\lambda}$ that almost all of the thick film region is normal. By forming composite substrates with only a few layers of cesium on top of gold, the effective adsorption potential can be tuned over a wide range [39]. Figure 5 shows the phase diagram of ${ }^{4} \mathrm{He}$ on 4.2 layers of cesium on gold, which has $T_{w}=1.5 \mathrm{~K}$. The prewetting line divides the $P-T$ plane into thick and thin film regions. The KT line divides the thick film region into super and normal parts; the transition line extends from $T_{\lambda}$ on the coexistence curve and terminates on the prewetting line in a critical endpoint 
near $T=2.05 \mathrm{~K}$, in accordance with theory [41]. Isotherms at temperatures above the critical endpoint show a prewetting step and then a KT transition with the same qualitative features as shown in Fig. 4. Below the critical endpoint, the film makes a direct transition from the thin normal state to a thick superfluid state, so prewetting and the superfluid transition are combined. This transition does not, however display the standard KT features. Because the film thickness is discontinuous, it never satisfies the relation of (1), so there is no dissipation peak; instead, the film is "born" superfluid without going through the KT transition.

Because of the absence of a quasi-solid layer, weak substrates offer the intriguing possibility of exploring superfluid transitions in submonolayer films at very low temperatures in direct contact with a metallic substrate. Cs is not well suited for this purpose because even at a thickness of a few atomic layers, the wetting temperature is so high that films at low temperature cannot be formed. Another way to strengthen the potential and lower the wetting temperature is to use the lighter alkalis rubidium, potassium, sodium and lithium as substrates. Our first attempts in this direction used rubidium. Rubidium is a slightly stronger adsorber than cesium, but the uncertainties in the potential parameters preclude a definitive prediction of whether there is a true wetting transition at coexistence [30]. Most of the existing data [40, 42] are consistent with a picture in which wetting takes place via a peculiar hysteretic transition that extends to the lowest temperatures, as shown in Fig. 6 (see however [44]). Although there does not appear to be a wetting temperature, the wet state is achieved via a first order prewetting-like transition that ends in a critical point near $T=2 \mathrm{~K}$. The superfluid transition extends from $T_{\lambda}$ at coexistence, but in contrast to cesium, joins the prewetting transition in a cusp at the critical point which is therefore a tricritical point. The precise intersection of a curve with a point seems unlikely without fine tuning of a parameter, but these types of tricritical intersections are common; a particularly famous example is the tricritical point for ${ }^{3} \mathrm{He}-{ }^{4} \mathrm{He}$ mixtures in which the lambda line hits the mixture coexistence curve at the mixing-demixing critical point which can be seen in Fig. 3.

Rubidium is apparently on the border between materials with a finite wetting temperature and those which wet at all temperatures. Its proximity to this border makes it particularly sensitive to the details of materials preparation and complicates the interpretation of the results. For the purposes of investigating submonolayer superfluidity, it would be useful to have a stronger substrate which helium decisively wets and a means of detecting superfluidity independently of the total coverage. Lithium has been proposed [43] as an ideal candidate for this study. The technology used for depositing films of cesium and rubidium (thermal evaporation from a resistively heated, infrared shielded oven) does not work well for lithium because the required temperatures are too high. We have implemented a pulsed laser deposition (PLD) technique which ablates a lithium target using nanosecond pulses of green light in vacuum at low temperature [45]. A typical isotherm of ${ }^{4} \mathrm{He}$ on lithium is shown in Fig. 7. In contrast to both cesium and rubidium, there is no sign of a prewetting step. On lithium, the helium film grows in a continuous way, with the thickness approximately proportional to the pressure. The slope of the linear part of the isotherm can be used to determine the binding energy, which is $13.7 \mathrm{~K}$ [45]. At $900 \mathrm{mK}$, there is a clear KT-type superfluid transition which occurs at a total thickness of approximately 
Fig. 7 Isotherm of ${ }^{4} \mathrm{He}$ on lithium, showing the frequency shift and the dissipation. The frequency shift is approximately linear in the pressure until the film goes superfluid near $P=3.5 \times 10^{-3}$ Torr; there is a corresponding peak in the dissipation. On the normal side of the transition, the coverage reaches 0.7 layers. On the superfluid side, the residual mass carried by the QCM is approximately 0.1 layers

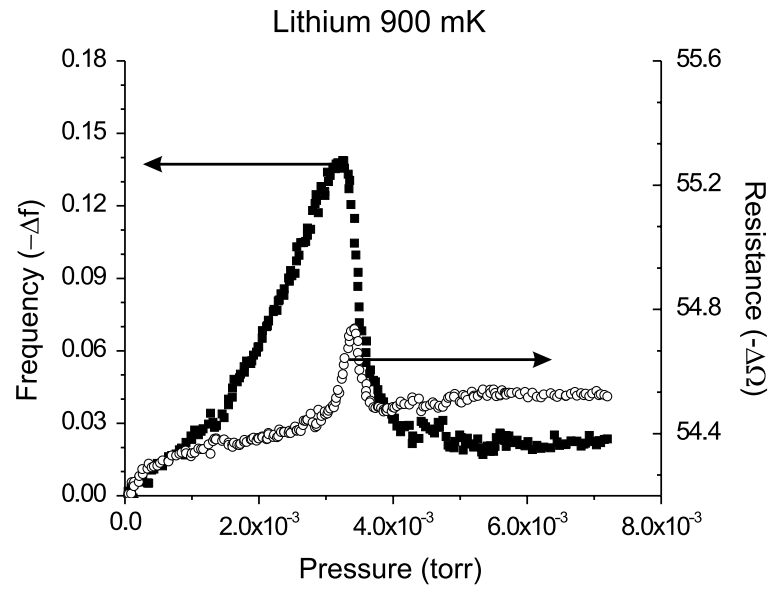

0.7 layers. On the superfluid side of the transition, there appears to be approximately 0.1 inert layers, which is smaller than on any other substrate. Even this small residual mass carried by the oscillator may be due to tortuosity rather than an intrinsic non-superfluid fraction.

\section{Future Work}

Our preliminary results indicate that the KT features seen in Fig. 7 disappear at lower temperatures. We believe that this is due to a collision of the KT line with the 2D liquid-vapor transition, which is expected for ${ }^{4} \mathrm{He}$ [46] but not ${ }^{3} \mathrm{He}$ [47]. This adds further support to the idea that lithium is a nearly ideal realization of $2 \mathrm{D}$ helium without the complications of a quasi solid layer. Just as for rubidium, when the superfluid transition becomes entangled with another phase transition, it is very helpful to have another diagnostic to make definitive assignments of phases. We plan to implement ellipsometry in our laser ablation apparatus so that the total coverage and the shear response can be measured simultaneously on the same surface. If these subsequent investigations confirm that helium on lithium is a close realization of $2 \mathrm{D}$ helium, it will be interesting to study ${ }^{3} \mathrm{He}-{ }^{4} \mathrm{He}$ mixture films. By increasing the ${ }^{3} \mathrm{He}$ concentration, it should be possible to depress the 2D liquid-vapor critical point to $T=0$ [48], which would be a novel system to study quantum critical behavior. Superfluidity in this system should also show a rich phase structure $[49,50]$.

Acknowledgements I would like to acknowledge stimulating conversations with Jim Rutledge, Lew Nosanow, Milton Cole, Will Saam, Jacques Treiner, Michael Schick, and my students David Ross, Jeff Phillips, Kalyani Sukhatme, Tim McMillan, Richard Lazarowich, Justin Burton, and Eli Van Cleve.

Open Access This article is distributed under the terms of the Creative Commons Attribution Noncommercial License which permits any noncommercial use, distribution, and reproduction in any medium, provided the original author(s) and source are credited. 


\section{References}

1. V.F. Kozhevnikov, M.J. Van Bael, P.K. Sahoo, K. Temst, C. Van Haesendonck, A. Vantomme, J.O. Indekeu, New J. Phys. 9, 75 (2007)

2. K.G. Sukhatme, J.E. Rutledge, P. Taborek, Phys. Rev. Lett. 80, 129 (1998)

3. W.F. Saam, J. Low Temp. Phys. (2009). doi:10.1007/s10909-009-9904-0

4. S.M. Gatica, M.W. Cole, J. Low Temp. Phys. (2009). doi:10.1007/s10909-009-9885-z

5. J.N. Israelachvilli, Intermolecular and Surface Forces (Academic Press, London, 1985)

6. E. Cheng, M.W. Cole, W.F. Saam, J. Treiner, Phys. Rev. Lett. 67, 1007 (1991)

7. P.J. Nacher, J. Dupontroc, Phys. Rev. Lett. 67, 2966 (1991)

8. K.S. Ketola, S. Wang, R.B. Hallock, Phys. Rev. Lett. 68, 201 (1992)

9. P. Taborek, J.E. Rutledge, Phys. Rev. Lett. 68, 2184 (1992)

10. J.E. Rutledge, P. Taborek, Phys. Rev. Lett. 69, 937 (1992)

11. T. McMillan, J.E. Rutledge, P. Taborek, J. Low Temp. Phys. 138, 995 (2005)

12. M. Schick, P. Taborek, Phys. Rev. B 46, 7312 (1992)

13. D. Ross, P. Taborek, J.E. Rutledge, Phys. Rev. B 58, R4274 (1998)

14. G. Mistura, H.C. Lee, M.H.W. Chan, J. Low Temp. Phys. 96, 221 (1994)

15. M.S. Pettersen, W.F. Saam, Phys. Rev. B 51, 15369 (1995)

16. M.S. Pettersen, W.F. Saam, J. Low Temp. Phys. 90, 159 (1993)

17. K.S. Ketola, R.B. Hallock, Phys. Rev. Lett. 71, 3295 (1993)

18. D. Ross, P. Taborek, J.E. Rutledge, Phys. Rev. Lett. 74, 4483 (1995)

19. D. Ross, J.E. Rutledge, P. Taborek, Phys. Rev. Lett. 76, 2350 (1996)

20. D. Ross, J.A. Phillips, J.E. Rutledge, P. Taborek, J. Low Temp. Phys. 106, 81 (1997)

21. D. Ross, J.E. Rutledge, P. Taborek, Science 278, 664 (1997)

22. D. Ross, P. Taborek, J.E. Rutledge, J. Low Temp. Phys. 111, 1 (1998)

23. J.E. Rutledge, D. Ross, P. Taborek, J. Low Temp. Phys. 113, 811 (1998)

24. J.M. Kosterlitz, D.J. Thouless, J. Phys. C 6, 1181 (1973)

25. M.P.A. Fisher, P.B. Weichman, G. Ginsteinand, D.S. Fisher, Phys. Rev. B 40, 546 (1989)

26. P.A. Crowell, F.W. Van Keuls, J.D. Reppy, Phys. Rev. B 55, 12620 (1997)

27. G.A. Csathy, J.D. Reppy, M.H.W. Chan, Phys. Rev. Lett. 91, 235301 (2003)

28. E. Cheng, G. Ihm, M.W. Cole, J. Low Temp. Phys. 74, 519 (1989)

29. V. Apaja, E. Krotscheck, M.D. Miller, J. Low Temp. Phys. 145, 369 (2006)

30. M. Boninsegni, L. Szybisz, Phys. Rev. B 70, 024512 (2004)

31. P.G. de Gennes, C.R. Acad. Sci. Ser. 2 B 327, 1337 (1999)

32. J.C. Burton, P. Taborek, J.E. Rutledge, J. Low Temp. Phys. 134, 237 (2004)

33. N. Hosomi, M. Suzuki, Phys. Rev. 77, 024501 (2008)

34. P. Mohandas, C.P. Lusher, V.A. Mikheev, B. Cowan, J. Saunders, J. Low Temp Phys. 101, 481 (1995)

35. J.G. Dash, Phys. Rev. Lett. 41, 1178 (1978)

36. M. Schick, O.E. Vilches, Phys. Rev. B 48, 9910 (1993)

37. M. Hieda, A.C. Clark, M.H.W. Chan, J. Low Temp. Phys. 134, 91 (2004)

38. P. Taborek, J.E. Rutledge, Phys. Rev. Lett. 71, 263 (1993)

39. E. Cheng, M.W. Cole, W.F. Saam, J. Treiner, J. Low Temp. Phys. 89, 739 (1992)

40. J.A. Phillips, D. Ross, P. Taborek, J.E. Rutledge, Phys. Rev. B 58, 3361 (1998)

41. W.F. Saam, J. Treiner, E. Cheng, M.W. Cole, J. Low Temp. Phys. 89, 637 (1992)

42. B. Demolder, N. Bigelow, P.J. Nacher, J. Dupont-Roc, J. Low Temp. Phys. 98, 91 (1995)

43. M. Boninsegni, M.W. Cole, F. Toigo, Phys. Rev. Lett. 83, 2002 (1999)

44. J. Klier, A.F.G. Wyatt, Phys. Rev. B 65, 212504 (2002)

45. E. Van Cleve, P. Taborek, J.E. Rutledge, J. Low Temp. Phys. 150, 1 (2008)

46. M.C. Gordillo, D.M. Ceperley, Phys. Rev. B 58, 6447 (1998)

47. R.C. Ramos, R.S. Ebey, O.E. Vilches, J. Low Temp. Phys. 110, 615 (1998)

48. R.C. Ramos, O.E. Vilches, J. Low Temp. Phys. 134, 55 (2004)

49. P.T. Finley, P.S. Ebey, R.B. Hallock, Phys. Rev. Lett. 98, 265301 (2007)

50. G.A. Csathy, M.H.W. Chan, Phys. Rev. Lett. 87, 045301 (2001) 